\title{
Significados das avaliações do ensino pelos estudantes: um estudo qualitativo na educação superior brasileira
}

\author{
Maria Beatriz Bettencourt \& Maria de Lourdes \\ Sá Earp
}

Resumo.

Este artigo apresenta resultados parciais de um estudo realizado em dez instituições de ensino superior (IES) sobre as avaliações do ensino feitas pelos estudantes (AEE), no âmbito do processo de auto-avaliação institucional prescrito pelo Sistema de Avaliação do Ensino Superior no Brasil. O presente estudo faz parte de uma pesquisa sobre os significados e efeitos das avaliações do ponto de vista dos estudantes. A metodologia utilizada é qualitativa e faz uso de entrevistas a estudantes que já tenham preenchido o questionário de avaliação institucional. A pesquisa revelou alguns fatores que influenciam as respostas dos estudantes: por um lado, a falta de confiança na confidencialidade das respostas e, por outro, uma crença positiva nos resultados de suas avaliações, ainda que seja reduzida a divulgação dos resultados e a participação dos estudantes ao longo do processo. Por fim, são feitas recomendações no sentido da institucionalização de uma cultura de avaliação formativa nas IES brasileiras.

Palavras-chave:

abordagem qualitativa; avaliação institucional; avaliação pelos estudantes; ensino superior. 


\section{Meanings of student evaluations of teaching: a qualitative research in Brazilian Higher Education}

Abstract: This article presents partial results of a study carried out in ten higher education institutions (HEI) on student evaluations of teaching (SET), within the scope of the institutional self-evaluation process prescribed by the Higher Education Evaluation System in Brazil. This study is part of a research on the meanings and effects of assessments from the student's point of view. The methodology employed is qualitative and makes use of interviews with students who have already completed the institutional evaluation questionnaire. The research revealed some factors that influence student responses: the lack of confidence in the confidentiality of the answers and, yet, a positive belief in the results of their evaluations, although results are rarely reported and student participation is reduced. Finally, the article includes recommendations to institutionalize a culture of formative assessment in Brazilian HEI.

Keywords: qualitative approach; institutional evaluation; Student Evaluation of Teaching (SET); higher education.

\section{Significado de la evaluación de la enseñanza por los estudiantes: una investigación cualitativa en la educación superior brasileña}

Resumen: Este artículo presenta los resultados de una investigación realizada en diez instituciones de educación superior (IES) centrada en las evaluaciones de educación por los estudiantes (EEE) dentro del proceso de autoevaluación institucional prescrito por el Sistema de Evaluación de la Educación Superior en Brasil. Este estudio forma parte de una investigación sobre los significados y los efectos de las evaluaciones del punto de vista de los estudiantes. La metodología es cualitativa y hace uso de las entrevistas con los estudiantes que ya han completado el cuestionario de evaluación institucional. La pesquisa reveló factores que influyen en las respuestas del estudiante: por un lado, la falta de confianza en la confidencialidad de las respuestas, por el otro, una creencia positiva en los resultados de las evaluaciones, aunque sea reducida la divulgación de los resultados y la participación de los estudiantes durante todo el proceso. Por último, se hacen recomendaciones sobre la institucionalización de una cultura de evaluación formativa en las IES brasileñas.

Palabras clave: enfoque cualitativo; evaluación organizacional; evaluación de la enseñanza por los estudiantes; enseñanza superior.

\section{Le sens des évaluations de l'enseignement par les étudiants: une étude qualitative dans l'enseignement supérieur brésilien}

Résumé: Cet article présente les résultats d'une recherche menée dans dix institutions d'enseignement supérieur (IES) sur les évaluations de l'enseignement faites par les étudiants, dans le cadre du processus d'auto-évaluation institutionnelle prévue par le Système d'Evaluation de l'Enseignement Supérieur au Brésil. L'étude présentée fait partie d'une recherche sur les significations et les effets de ces évaluations, du point de vue des étudiants. La méthodologie est qualitative et fait appel à des entrevues avec des étudiants ayant déjà complété le questionnaire d'évaluation institutionnelle. L'enquête a révélé des facteurs qui influent sur les réponses des étudiants, tels que d'une part, le peu de confiance dans la confidentialité des réponses et, d'autre part, une croyance positive dans les résultats de ces évaluations, même si la diffusion des résultats et la participation des étudiants dans le processus sont réduites. Pour terminer, nous présentons des recommandations concernant l'institutionnalisation d'une culture d'évaluation formative dans les IES brésiliennes.

Mots-clés: approche qualitative; évaluation institutionnelle; évaluation de l'enseignement par les étudiants; enseignement supérieur. 


\section{Introdução}

A AEE é uma realidade consolidada nos EUA e no Canadá, onde se pratica há mais de meio século (Hativa, 2008). Esta prática social tem o propósito de fornecer informação, fundamentalmente ao professor e à administração, com o fim de ser utilizada na melhoria do ensino e na gestão da carreira dos docentes.

Acresce que, no referido contexto, se generalizou a utilização de um questionário, o Students Evaluation of Educational Quality (SEEQ), que facilitou a realização de pesquisas psicométricas para testar a sua confiabilidade e validade e apurar que variáveis afetam os seus resultados.

Numerosas pesquisas (só na base de dados Eric podemos encontrar cerca de 3.000) tiveram por objeto determinar quais os fatores que influenciam as respostas dos estudantes e medir o enviesamento dos resultados em função de características:

- Das disciplinas - dificuldade, carga de trabalho exigido ao estudante;

- Dos professores - sexo, idade, aparência, grupo étnico, experiência profissional;

- Dos estudantes - sexo, expetativas, motivação, assiduidade, esforço dispendido;

- Administrativas - número de alunos por turma e de turmas por professor, formas de administração dos questionários.

As pesquisas apontam que muitos destes fatores têm alguma influência na resposta dos estudantes; foi possível, por exemplo, verificar algum viés associado a características do professor, como sexo, idade, grupo étnico, que refletem discriminações e preconceitos existentes na sociedade.

Como prática social, a AEE tem sido objeto de crítica por muitos professores que a consideram pouco adequada e mesmo nociva, por ter como efeito possível a inflação das classificações dos estudantes (Davidovich e Soen, 2009). Assim, a hipótese de que a avaliação que os estudantes fazem do ensino seja um reflexo das classificações que obtiveram na disciplina tem também sido testada em numerosos estudos. De fato, várias investigações comprovam a existência de uma correlação entre as AEE de uma disciplina e as classificações que os estudantes nela obtêm (Howard e Maxwell, 1982; Marsh, 1987; Krautman e Sander, 1999). Contudo, esta correlação é moderada (média de .20, segundo Davidovich e Soen, 2009) e a literatura não permite a validação da hipótese, pois são nela são apontadas explicações contraditórias (d'Appolonia e Abrami, 1997).

Estudos posteriores, utilizando modelos com variáveis múltiplas, têm sido um pouco mais conclusivos. Por exemplo, Wright e Palmer (2006) testaram um modelo que incluiu as variáveis motivação e trabalho desenvolvido pelos estudantes e suas classificações como fatores influenciando a AEE e que se revelou extremamente compatível com os dados recolhidos. 
Para além da diversidade de fatores relacionados com o estudante, a atividade de ensinar é, também ela, uma atividade multifacetada. Os questionários de AEE não podem medir todas as suas dimensões; por exemplo, o instrumento SEEQ abrange nove fatores ou componentes, criados a partir de uma análise lógica do trabalho de ensinar e que são: "Instrutor enthusiam, Breadth of coverage, Organisation/Clarity, Workload/ Difficulty, Assignements/Readings, Learning/Value, Examination/Grading, Group interaction, Individual Rapport" (Marsh, 2007, 323). Mas outras categorizações existem, correspondentes a outras concepções do que é um ensino eficaz. As diferentes visões de qualidade de ensino e as diversas formas de as operacionalizar dão origem a uma multiplicidade de questionários de AEE, o que, em si mesmo, não constituiria um problema se todos tivessem um escopo clarificado.

São complexos os fatores que influenciam as AEE, pois incluem o docente, o estudante, as condições de interação e condições externas; é, contudo, necessária a sua compreensão para se poder utilizar as AEE de forma adequada. Diversos autores têm ressaltado a necessidade de triangular os resultados das AEE com outros indicadores (taxas de sucesso, avaliação externa dos programas, empregabilidade dos diplomados, avaliação pelos diplomados), porque só uma abordagem multifacetada permite avaliar a qualidade do ensino universitário e este visa outros objetivos sociais, para além da satisfação dos estudantes (Abernot, Y, et al, 2013).

Com todas estas ressalvas, as pesquisas têm evidenciado que as AEE são válidas enquanto medidas de qualidade do ensino, em muitos dos seus aspetos, tendo-se verificado elevada correlação entre avaliações realizadas por meio de questionários diferentes e mesmo entre avaliações feitas por estudantes e por colegas professores (Romainville, 2004).

Embora muito numerosas, as pesquisas sobre a AEE têm-se focalizado, sobretudo, na sua representatividade, validade e fidelidade, enquanto avaliação.

Os efeitos das AEE na melhoria do ensino, na profissão docente, institucionais e sociais têm sido pouco pesquisados. Os trabalhos de Younès (2015) têm procurado distinguir as AEE segundo a sua eficácia na melhoria do processo de ensino. Na perspectiva da autora, a AEE é uma regulação individual e coletiva que se alimenta de diferentes formas de partilha, nos momentos em que os variados atores da IES constroem sentidos conjuntos. Ao longo do processo de AEE, Younès identifica cinco formas de partilha que se identificam com as culturas locais:

1. Partilha de responsabilidades na realização de todo o processo;

2. Partilha ex-ante na construção dos critérios de qualidade do ensino;

3. Partilha ex-ante na elaboração do questionário;

4. Partilha ex-post dos pontos de vista de professores e estudantes;

5. Partilha ex-post na análise dos resultados das AEE e na preparação de decisões subsequentes. 
O efeito transformador da cultura universitária que, em alguns casos, decorre do processo de AEE, é analisado pela autora. No mesmo sentido, o estudo de Bettencourt (2005) permite acompanhar o impacto do processo de avaliação institucional na cultura das instituições de ensino superior (IES).

Também tem sido pouco questionado na literatura desta área o significado das narrativas a que se reportam as respostas estudantis. A presente pesquisa propõe-se tratar as AEE como discursos com potencial transformador da cultura e das representações do ensino superior.

\section{A AEE no contexto brasileiro}

A partir dos anos 80 do século passado, as AEE passaram a ser um instrumento de regulação dos sistemas de ensino superior, no contexto das práticas gestionárias da Nova Gestão Pública (NGP), visando a instauração de um controle de tipo ex-post, conjugando o autocontrolo institucional com a avaliação pelos resultados (Finger et Ruchat, 1997). Neste sentido, as AEE são utilizadas por instituições de acreditação em todo o mundo e, em alguns países, também pelos governos centrais (caso do Brasil, do Reino Unido e dos Países Baixos).

No Brasil, tal como em muitos países, a realização de avaliações do ensino pelos estudantes é uma prática inscrita no processo de auto avaliação institucional que todas as instituições de ensino superior devem levar a cabo, no âmbito de sistemas diversificados de avaliação e regulação do ensino superior. É esperado que do processo de AEE resulte uma auto regulação interna das instituições e uma melhoria da qualidade do ensino. Na conceção das políticas públicas, o governo federal brasileiro definiu que a avaliação é o referencial básico e norteador para a regulação e supervisão da educação superior (Júnior et al, 2014), colocando as IES no papel de sujeito da avaliação, visto o Plano de Desenvolvimento Institucional (PDI) ser o ponto de partida do processo.

De acordo com esta orientação, o Sistema Nacional de Avaliação da Educação Superior brasileiro (SINAES) permite que as instituições decidam, com alguma margem de autonomia, o modo como os estudantes realizam a avaliação do ensino. Como resultado dessa autonomia, existe grande diversidade nas modalidades de avaliação escolhidas, nos instrumentos utilizados e nas formas adotadas para divulgação e uso dos resultados. Uma enorme massa de informação é recolhida nos questionários respondidos por estudantes (e também por professores e funcionários) e são milhares as horas empregues no tratamento desses dados. É esperado que deste trabalho resulte uma autoregulação interna das instituições, bem como o desenvolvimento de uma cultura de avaliação que leve as IES a planejar o futuro em função dos resultados obtidos. 


\section{Metodologia da pesquisa}

Para melhor compreender as AAE torna-se importante investigar as condições concretas em que os estudantes respondem aos questionários, as circunstâncias diversas que condicionam as suas respostas, a importância que eles Ihes atribuem e o que pensam sobre os efeitos da AEE na instituição. Nesse sentido, no âmbito da pesquisa, pretende-se responder às seguintes questões:

1. Como os estudantes participam da avaliação do ensino? Quais os procedimentos adotados e como os estudantes reagem?

2. Que condicionantes influenciam as respostas dos estudantes e quais os seus significados, nas IES estudadas?

3. Como é que as AEE são localmente apropriadas e que efeitos têm na vida institucional e na cultura das IES?

A escolha da abordagem qualitativa decorre da própria natureza da pesquisa, em que se pretende conhecer o como e o porquê das ações dos agentes envolvidos no processo de AEE. A metodologia escolhida é a do estudo de caso instrumental e múltiplo. Adotando a perspectiva de Stake (1995), consideramos este estudo de caso como instrumental, pois o caso desempenha o papel de suporte para o estudo em profundidade de um outro fenómeno, neste caso, as AEEs, suas condicionantes políticas e institucionais e seus efeitos. Trata-se também de um estudo de caso múltiplo (Yin, 1984), pois os locais estudados são vários, não porque se pretenda generalizar os resultados, mas sim para que a análise incida sobre realidades diferenciadas que, pela sua variedade, permitam compreender melhor o fenómeno estudado.

A pesquisa foi desenvolvida em nove IES do Estado do Rio de Janeiro - três públicas e seis privadas - e uma IES privada do Estado de Minas Gerais. Cada local foi estudado separadamente e numa fase posterior será feita uma análise comparativa.

Cabe registrar que não se pretendeu constituir uma amostra representativa das IES brasileiras, mas sim reunir um conjunto variado e equilibrado de instituições que permitissem analisar o objeto de estudo na sua complexidade. Assim, para nós, o estudo de caso foi, fundamentalmente, uma estratégia de pesquisa que se socorreu de outros métodos, como a observação de campo, a análise documental e as entrevistas a dirigentes e estudantes.

Como o campo de investigação são Instituições de Ensino Superior (IES), tipo de organização que é familiar a todos os envolvidos na pesquisa, procuramos construir um outro olhar, investigativo e interrogativo, sobre uma realidade que se acredita conhecer bem, em função da vivência cotidiana dos membros da equipe de investigação, como estudantes e/ou professores de IES. Nesse sentido espera-se que todos realizem a tarefa de "estranhar o familiar". Entretanto, como afirma Velho (1978) [2], "o que 
sempre vemos e encontramos pode ser familiar, mas não necessariamente conhecido" (p. 126, grifos no original).

A primeira etapa de trabalho centrou-se na observação de campo. Este tipo de observação pareceu-nos o mais adequado para que se compreenda o contexto institucional, pois responde às necessidades de:

\begin{abstract}
"descrever e compreender o modo como as pessoas vivem, trabalham e se relacionam num determinado contexto social, implicando que o investigador se insira na situação (...) e observe o próprio contexto, os padrões das relações entre as pessoas, o modo como reagem aos eventos que ocorrem..." (Cozby, 1989, 48).
\end{abstract}

Cabe comentar que o trabalho de campo na IES é antecedido por um estudo do site da instituição, no sentido de procurar os caminhos e interfaces para se chegar às informações sobre avaliação institucional (Al). Nessa navegação procuramos perceber como a Al se apresenta no site, que informações sobre o processo estão disponíveis e que resultados estão publicados.

Em seguida, a equipe de pesquisa realiza as visitas de observação nas IES, munida de cadernos de campo para registro de informações sobre as instituições, tais como: descrições dos espaços físicos, maneiras como os estudantes utilizam esses espaços, diálogos, cenas e conflitos, enfim, informações sobre o que os atores fazem, o que pensam sobre o que fazem, o que falam sobre o que fazem (Malinowski, 1978). No exercício desse tipo de investigação, tanto ouvir quanto olhar não podem ser tomados como faculdades totalmente independentes: olhar por si só não seria suficiente, o ouvir complementa o olhar (Sá Earp, 2012). Um momento importante dessa fase inicial de exploração é a discussão e análise dos cadernos de campo de todos os membros da equipe de pesquisa. As observações são compartilhadas no sentido de que o olhar de cada investigador seja ampliado pelos olhares dos demais. Como sugere Vianna (2003), diversas pessoas a partir de suas experiências individuais podem ver o mesmo objeto de formas diferentes. O objeto é o mesmo, o que muda é a organização mental de quem observa.

Deve-se registrar que nessas observações o investigador faz contato com uma pessoa da IES ligada ao processo de avaliação institucional, que será posteriormente entrevistada a partir de um roteiro previamente elaborado. No final desta etapa, é feita a redação do relatório de campo, que pode incluir imagens.

Na segunda etapa, é realizada uma entrevista a um membro da CPA da IES, ou a algum membro da equipe de direção ou coordenação, no caso de serem estes os órgãos responsáveis pelo processo de Al. Nessa entrevista pretende-se conhecer, do ponto de vista do entrevistado, como se desenvolveu o processo de avaliação 
institucional, como foram elaborados e aplicados os instrumentos e o que é feito com os resultados das avaliações. Cabe registrar que a realização desse tipo de entrevista passa por todas as providências necessárias, apontadas por outros pesquisadores (Silva, 2012): os contatos prévios, o cuidado com a marcação, a escolha do local e a postura do entrevistador, entre outras.

Em seguida, entra-se no fulcro do trabalho: a entrevista aos estudantes das IES. Um aspecto particularmente importante é assegurar a confidencialidade das respostas. Assim, ao apresentar-se, o entrevistador esclarece o propósito do estudo, indicando a instituição a que se vincula e garante o sigilo das informações recolhidas. Os entrevistados são estudantes que responderam, previamente, ao questionário institucional (Qul). Essa entrevista destina-se a saber como os entrevistados responderam às questões desse instrumento, como as compreenderam e se dariam ou não a mesma resposta nas circunstâncias atuais e quais as razões. No roteiro existem perguntas comuns aos estudantes de todas as IES: qual a sua opinião sobre a importância destes questionários, quais os efeitos que deles esperam e quais os reais impactos da avaliação. Outra parte do roteiro de entrevista é elaborada a partir do questionário de cada instituição. Em cada IES foram entrevistados pelo menos dois estudantes e as transcrições estão identificadas por E1 e E2.

Constatamos que o fato do entrevistador ser uma pessoa fora do círculo de relações do informante e externa à instituição facilitou uma maior abertura por parte dos entrevistados. A garantia de que o pesquisador "entra, mas sai" cria situação de menor compromisso do que aquela existente com pessoas mais próximas e de que têm resultado diálogos com uma comunicação fluida e interessada por parte dos entrevistados.

Em termos de tratamento de dados, tínhamos a intenção de nos basear na classificação de Younès (2015), já apresentada, relativa às diferentes formas possíveis de participação dos estudantes na avaliação do ensino.

Contudo, em nove dos dez casos estudados, os estudantes não participam da construção dos critérios de qualidade do ensino, tampouco da elaboração do questionário, da análise dos resultados das $\mathrm{AEE}$ ou da preparação de decisões subsequentes. Ainda que sejam atores fundamentais no processo, a sua participação reduz-se ao prenchimento dos questionários de Al. Só num caso (IES 7) existe uma partilha de responsabilidades com os estudantes na execução da Al: os estudantes de Métodos de Pesquisa administram o questionário, fazem o tratamento de dados e elaboram relatórios, mas não são chamados para a definição de critérios de avaliação nem para a construção dos questionários.

Após uma leitura exaustiva (Minayo, 2006) do material levantado, elaboramos grandes categorias que pudessem dar resposta às questões da investigação e que servissem de eixo para a análise dos dados, a saber:

- Motivações dos estudantes para responder ao QAl; 
- Mudança de resposta aos itens do QAI e suas razões;

- Divulgação junto dos estudantes dos resultados da Al;

- Efeitos da Al sob o ponto de vista estudantil.

\section{Resultados da pesquisa}

É a partir das falas dos entrevistados que podemos compreender os cenários em que se desenrola a AEE nas IES brasileiras, os papéis que os estudantes assumem no processo e o que dele pensam. Os resultados aqui apresentados são ainda parciais, pois a fase de análise não terminou, estando a ser objeto de trabalho do grupo de pesquisa.

Como foi indicado, na maioria das IES estudadas, a participação dos estudantes limita-se ao preenchimento de um questionário. Encontramos duas formas dessa participação. A maioria deles chegou aos questionários por iniciativa própria e poucos por ser obrigatório. Constatámos que nas duas IES (1 e 2) em que o preenchimento dos questionários é obrigatório, as taxas de resposta atingem os 100\%. Nestes dois casos, o estudante só tem acesso à sua conta depois de preencher em linha o questionário. As taxas de resposta são muito diferentes quando a resposta é facultativa, situando-se entre $1,5 \%$ e $20 \%$.

Quando a sua participação é facultativa, os estudantes respondentes revelaram, como motivações para a resposta, a vontade de exprimir uma reclamação, mais ou menos pontual, junto à administração:

Entrevistador: "Se não é obrigatório, porque você respondeu?"

Estudante: "É porque o questionário, prá gente, é uma forma de instrumento de avaliação do que a gente vive. Como se fosse o nosso poder de reclamar de algo por um instrumento oficial; se os professores são satisfatórios, se a infraestrutura está satisfatória... A forma que a gente tem de reclamar e se tornar um dado e esse dado ser visto por quem gerência a parte administrativa da faculdade e que isso possa ser resolvido" (IES 3: E1).

Estudante: "Porque, na época, a gente... era no começo da faculdade, tinha alguns professores que a gente reclamava muito; porque eram professores que a gente discordava, pelo menos eu discordava de alguns pontos; então, como tinha uma pesquisa eu me senti na obrigação de pôr e esclarecer o porquê daquela minha satisfação. Essa foi a maneira de eu reclamar algum comportamento ou método" (IES 5: E1).

A mudança de opinião dos estudantes entrevistados sobre itens do QAI tem uma expressão diferente nas IES em que a resposta ao QAI é obrigatória e naquelas em que é facultativa. Assim, nas duas IES em que a resposta ao QAI é obrigatória, são expressivas as diferenças entre as respostas que os estudantes deram no questionário institucional e as opiniões, muito menos positivas, que, posteriormente, expressaram na entrevista. Na IES 1, das 14 questões do QAI selecionadas para a entrevista, em 
$60 \%$ das respostas existe divergência, sendo que o sentido dominante da mudança é o da diminuição da classificação: só em dois itens a avaliação se mantém e, em três itens, ela melhora.

Associada a essa mudança, surgiu nas entrevistas a questão da possível identificação do respondente, não prevista na pesquisa, mas vista como uma ameaça real pelos quatro entrevistados:

"Existem nas instituições profissionais sem ética: um professor disse na sala de aula que uma aluna o tinha avaliado mal e que ela ia se dar mal" (IES 2: E1). Segundo outro aluno, as respostas na entrevista são diferentes porque "finalmente posso falar a verdade sobre a minha opinião" (IES 2: E2).

A sugestão dos estudantes é a de "publicar a Avaliação Interna em um ambiente virtual próprio, desvinculado do Campus Virtual, de modo a que o respondente possa ter, se o desejar, o seu anonimato preservado e (...) sinta-se confortável para expressar as suas opiniões livremente e sem receio de represália" (IES1: E1). De preferência, "seria apropriado que a AI não fosse realizada em período próximo a provas" (IES1: E2).

Com exceção destas duas IES, a mudança de opinião dos entrevistados é pontual, incidindo em aspetos em que o aluno acha que houve mudança na IES ou na sua forma de encarar a questão.

Como foi referido, em nenhuma IES os alunos foram chamados a partilhar com outros atores os momentos de construção dos critérios de qualidade do ensino ou da elaboração dos questionários. Foi também verificado, em várias IES, que o empenho institucional na divulgação do processo da Al e em levar os estudantes a responder aos questionários foi reduzido. Em muitos casos, o QAl chega ao estudante unicamente através do site da instituição ou por correio eletrônico:

"Na hora em que entrei no site da faculdade apareceu a mensagem: 'você precisa responder ao questionário'. Nenhum professor falou desse questionário em específico. Não me lembro de ouvir em sala de aula. Só na internet" (IES 7, E2).

"Eu recebi por email da aula, da universidade, se eu gostaria de responder... no email eles davam um link. Você entrava na página do questionário e se cadastrava" (IES 4, E2).

Mesmo quando existem lugares próprios para afixar notícias da CPA, esses espaços não são utilizados, como no caso da IES 8, onde se observou um mural vazio em que estava escrito "Avaliação Institucional".

Em contrapartida, em três IES, existiu um investimento da Direção no sentido de obter a colaboração dos alunos no preenchimento do QAI, como na IES 10, em que um estudante diz: "Eles (direção) vêm nas aulas e nos chamam para preencher o questionário...falam que é importante a nossa participação" (IES 10: E1). Nestas IES, além da intervenção da direção ou de elementos do pessoal, também foram afixados cartazes, banners e outros meios de alertar os estudantes. 
Na divulgação dos resultados da Al também existe uma grande variedade de situações: em dois casos (IES 1 e IES 9), são inseridos no site institucional alguns resultados escolhidos; noutros dois casos os bons resultados são utilizados pelas IES no seu marketing, inserido no site institucional (IES 1) ou nos folhetos da Universidade e dos cursos (IES 8). Só uma instituição realiza reuniões para divulgação de resultados, mas que são unicamente frequentadas por professores. A situação predominante é a de não existir nenhuma divulgação dos resultados da Al.

Os estudantes entrevistados são quase unânimes em declarar que não conhecem os resultados das AEE e que não perceberam qualquer impacto em termos de mudança na instituição. A fala a seguir exemplifica esse modo de pensar: "Acredito que os resultados não tenham sido utilizados porque a faculdade continua a mesma desde que entrei" (IES7).

Algumas direções referem ter realizado melhorias nas instalações e nos equipamentos motivadas pela avaliação dos estudantes (IES 3 e 9). Em alguns casos os estudantes associam essas mudanças à Al: "algumas salas não tinham ar condicionado e depois da avaliação institucional foram colocados em todas as salas" (IES 8, A1). Não foram sinalizadas situações de utilização das AEE para efeitos de melhoria das atividades de ensino e aprendizagem e, no entendimento dos alunos, as avaliações que realizam não têm efeitos significativos: "Não, não.. nunca teve. Eu já tinha feito, desde a época do ciclo básico, já tinha feito estes questionários, nunca ouvi falar desses resultados" (IES 6, A2).

Cabe referir que muitas respostas evidenciam o sentido crítico dos estudantes entrevistados sobre o processo avaliativo, apontando algumas incorreções, inclusive na construção do questionário.

\section{Considerações Finais}

Da análise do material recolhido, pudemos levantar algumas hipóteses interpretativas que configuram diferentes formas de pensar a avaliação institucional.

Um conjunto de estudantes tem suas respostas marcadas pela desconfiança quanto a aspectos fundamentais do processo, como o respeito pela confidencialidade das respostas. De maneira geral, essa crença se apresenta de forma mais intensa em estudantes de instituições em que é obrigatório participar na avaliação institucional, respondendo ao questionário. Outra tendência nas respostas dos estudantes aponta para uma crença, digamos, mais "positiva" na avaliação institucional, evidenciada pelo condicionamento das respostas dos estudantes em função das repercussões que elas têm na classificação das IES e, logo, para os seus diplomados. Alinhados a essa tendência também podemos colocar os estudantes que participam de forma espontânea das avaliações. 
Acreditando, mais ou menos, na avaliação institucional, os entrevistados demonstram falta de confiança no seu impacto, pois não se dão conta do seus efeitos reais.

É recomendável que os estudantes sejam chamados a participar em outras fases do processo de avaliação. A participação dos estudantes na construção e validação dos questionários é desejável e contribui para a melhoria do processo, assim como a sua intervenção na discussão dos resultados que irá implicar a existência de impacto a nível pedagógico.

A realização da AEE assume os contornos de uma formalidade rotineira nos processos de avaliação institucional estudados, sem que dela tenham resultado melhorias no ensino. Em vez de se instaurar uma cultura avaliativa de auto-regulação institucional, instala-se um ritual de avaliação em que são utilizados instrumentos e procedimentos avaliativos, porque assim está determinado. É uma cultura de conformação às regras que se sabe serem objeto de supervisão, mas não está sendo criada uma cultura de auto regulação institucional sistemática, visando a melhoria, a partir dos resultados da avaliação. O que se passa é que "(...) a despeito das IES poderem manifestar-se contra o processo burocrático que envolve as políticas públicas no seu setor, é cômodo para elas aceitar o processo, uma vez que não envolve mudanças profundas, complexas e delicadas com rebatimentos práticos em seu cotidiano, (...)" (Silva Júnior et al, 2014, 235). Como os dirigentes não fazem uma utilização sistemática dos resultados da avaliação na sua gestão corrente, eles estão também pouco sensibilizados para a importância de promover a participação dos estudantes em todo o processo e, em especial, na discussão dos seus resultados.

Assim, nas IES estudadas, ainda que o processo de AEE possa ter tido alguns efeitos em termos de regulação interna, como por exemplo na definição de prioridades na aquisição de equipamentos, não detectamos evidências, até agora, de utilização da avaliação para uma melhoria da qualidade do ensino. Em nosso entender, essa fraca rentabilidade pedagógica das AEE deve-se, a nível das IES, a não se terem criado os espaços colaborativos entre professores, responsáveis, estudantes e outros atores externos (antigos alunos, empregadores, lideres culturais e sociais) destinados à reflexão conjunta (seguida de decisão) sobre os finalidades do ensino, no curso e nas disciplinas, sobre a eficiência dos percursos formativos e sobre aquilo que, segundo os contextos, pode melhorar ou dificultar a aprendizagem.

A nível do contexto nacional de realização da AEE, a sua utilização na avaliação externa e nos rankings não pode deixar de ter influência nos processos e nos resultados. A consolidação dos processos de avaliação das IES seria facilitada se a essa avaliação não estivessem associados aspectos classificatórios, com repercussões penalizadoras.

A institucionalização de uma cultura de avaliação formativa deveria ser uma prioridade das políticas institucionais e também das políticas nacionais de ensino superior. 
Por isso somos da opinião de Silva Júnior et al $(2014,235)$ quando dizem que a mudança deve passar pela necessidade de mobilização da sociedade para questionar esse modelo de regulamentação, para que a causa educacional, em termos de qualidade dos serviços prestados pela IES públicas e privadas, passe do discurso e se efetive.

\section{Referências Bibliográficas}

Abernot, Y., Gangloff-Ziegler, C. \& Weisser, M. (2012). Contribution à l'épistémologie de l'évaluation des enseignements par les étudiants. Éducation et socialisation [Em linha], 32| 2012, disponível em: http://edso.revues.org/361 consultado a 06 maio 2015.

Abrami, P. C., d'Apollonia, S. \& Rosenfield, S. (2007) The Dimensionality of Student Ratings of Instruction: What We Know and What We Do Not. In: Perry, R. \& Smart, J. C. The Scholarship of Teaching and Learning in Higher Education: An Evidence-Based Perspective, (319-383). New York: Springer.

Bettencourt, B. (2005). L'évaluation des établissements en tant que mode de régulation. Une étude de cas en enseignement collégial. Tese de Doutoramento em Ciências da Educação. Montréal: Université de Montréal.

Cosby, P. (1989). Methods of behavioral research. Mountain View, CA: Mayfield Publishing.

D’Appolonia, S. \& Abrami P. C. (1997). Navigating student ratings of instruction. American psychologist, 52 (11), 1198-1208.

Davidovitch, N. \& Soen, D. (2009). Myths and facts about student surveys of teaching the links between students' evaluations of faculty and course grades. Journal of College Teaching and Learning, 7 , (VI), 41-49.

Finger, M. et Ruchat, B. (1997). Le New Public Management: État, administration et politique. Pour une nouvelle approche du management public. Réflexions autour de Michel Crozier, (pp. 34-56). Paris: Seli Arslan, 34-56.

Galbraith, C., Merrill, G. \& Kline, D. (2011). Are Student Evaluations of Teaching Effectiveness Valid for Measuring Student Learning Outcomes in Business Related Classes? A Neural Network and Bayesian Analyses. Research in Higher Education, 1, 1-22.

Hativa, N. (2008). Myths and facts about evaluation surveys by students, Al-Agova, 7, 13-14.

Howard, G. S. \& Maxwell, S. E. (1980). Correlation between student satisfaction and grades: a case of mistaken causation? Journal of Educational Philosophy, 72(4), 810-820.

Malinowski, B. (1976). Argonautas do pacifico ocidental. São Paulo: Editora Abril.

Marsh, H. W. (2007). Students' evaluations of university teaching: A multidimensional perspective. In R. P. Perry e J C. Smart (Ed.) (2007). The Scholarship of Teaching and Learning in Higher Education: An Evidence-Based Perspective (pp. 319-384). New York: Springer. 
Marsh, H. W. (1987). Students' evaluations of university teaching: Research findings, methodological issues, and directions for future research. International Journal of Educational Research, 11(3), 253-388.

Penny, A. R. \& Coe, R. (2004). Effectiveness of Consultation on Student Ratings Feedback: A MetaAnalysis. Review of Educational Research, 74 (2), 215-253.

Richardson, J. T. E. \& Slater, J. (2007). The National Student Survey: Development, findings and implications. Studies in Higher Education, 32 (5), 557-580.

Romainville, M. (2004). Esquisse d'une didactique universitaire. Disponível em: http://www.det.fundp. ac.be/spu/ Consultado em 2/10/2015.

Sá Earp, M. L. (2012). Observação. In: Elliot, L. (2012). Instrumentos de Avaliação e Pesquisa (193-227). Rio de Janeiro: Wak Editora.

Silva Júnior, A., Polizei, Caio E. G., Souza, S., Silva, P de O. M. \& Silva, S.P. (2014). Políticas públicas para a educação superior: a avaliação, a regulação e a supervisão de IES privadas em debate. Ensaio: Avaliação e Políticas Públicas Educacionais, 82, (XXII), 215-240.

Spooren, P., Brockx, B. \& Mortelmans, D. (2013). On the validity of student evaluation of teaching: The state of the art. Review of Educational Research, 83 (4), 598-642.

Spooren, P., Mortelmans, D. \& Thussen, P. (2012). 'Content' versus 'style': acquiescence in student evaluation of teaching? British Educational Research Journal, 38 (1), 3-21.

Stake, Robert E. (1995). The art of case study research. Thousand Oaks, California: SAGE Publications, Inc.

Velho, G. (1978). Observando o familiar. In Nunes, E. de O. (Org). A aventura sociológica: objetividade, paixão, improviso e método na pesquisa social. (pp. 36-46). Rio de Janeiro: Zahar.

Vianna, H. (2003). Pesquisa em educação: a observação. Brasília, DF: Plano Editora.

Yin, Robert K. (1984). Case study research. Design and methods. Beverly Hills, California: SAGE Publications, Inc.

Younès, N. (2015). L'évaluation de l'enseignement par les étudiants: le tournant du partage. Evaluer. Journal International de Recherche en Education et Formation, 1(1), 79-90.

Wright, R. \& Palmer, J. (2006). A comparative analysis of different models explaining the relationship between instructor ratings and expected student grades. Educational Research Quarterly, 30 (2), 3-18. 
Maria Beatriz Bettencourt

Professora do Mestrado em Avaliação, Fundação CESGRANRIO, Rio de Janeiro, Brasil

Email: beatrizbettencourt@yahoo.com

Maria de Lourdes Sá Earp

Professora do Mestrado em Avaliação,

Fundação CESGRANRIO, Rio de Janeiro, Brasil

Email: malusaearp@gmail.com

Correspondência

Maria Beatriz Bettencourt

Rua Santa Alexandrina, 1100 - Rio Comprido, Rio de Janeiro

RJ, 20261-232, Brasil

Data de submissão: Julho 2016

Data de avaliação: Outubro 2016

Data de publicação: Julho 2017 